\title{
Más allá de la biodiversidad: aportes de la antropología a la conservación marina en Chile*
}

\begin{abstract}
Beyond biodiversity: contributions of anthropology to marine conservation in Chile
\end{abstract}

\section{Francisco Araos ${ }^{* *}$}

\section{Resumen}

En los últimos años, la Conservación Marina se ha transformado en un asunto clave en el campo ambiental chileno. Impulsado por las reformas políticas y el cumplimiento de los acuerdos internacionales, Chile ha logrado expandir el número y cobertura de las Áreas Marinas Protegidas. Sin embargo, este proceso ha puesto en evidencia las transformaciones que las áreas protegidas han provocado en las poblaciones locales, revelando la importancia de

Este trabajo recibió apoyo financiero y forma parte del proyecto CONICYT /FONDECYT/PROYECTO No 3150341.

** Programa Atlas, Departamento de Ciencias Sociales, Universidad de Los Lagos. Avda. Fuchslocher 1305, Osorno.Nº 72130323. incorporar la dimensión humana en la gestión de la biodiversidad. El artículo tiene por objetivo presentar este nuevo tema de investigación en el campo socio-ambiental chileno, exponiendo los principales aportes de la antropología en el contexto internacional y el estado actual de esta discusión en el país. A la luz de este debate se presentan los elementos claves de una propuesta analítica para el estudio de las dimensiones humanas de la conservación marina y sus contribuciones para las políticas públicas.

Palabras clave: Conservación Marina, Dimensiones Humanas, Antropología, Políticas Públicas.

\begin{abstract}
Over the last years, Marine Conservation became a key issue in the environmental field in Chile. Promoted by the current environmental political reforms and the compliance of the international biodiversity targets, Chile increased the number and coverage of Marine Protected Areas. Nevertheless, the creation of protected areas has produced transformations on local communities, revealing the importance of incorporating the human dimension in the management of biodiversity. This paper aims at presenting this new research theme for the socioenvironmental field in Chile, offering an overview of the main contributions of anthropology in the international context and the current discussion in the country. Based on this debate the paper explores the key issues of an analytical framework for the study of the human dimensions of marine conservation and its contributions for public policies.
\end{abstract}

Key words: Marine Conservation, Human Dimensions, Anthropology, Public Policies. 


\section{Introducción}

La preocupación por la conservación de la biodiversidad es reciente entre los estudios antropológicos. A pesar de que el interés de la antropología por las relaciones entre los grupos humanos y la naturaleza es antiguo $y$ ha sido objeto de estudio de connotados antropólogos de diversas épocas y escuelas, la conservación de la biodiversidad como problema antropológico emergió solamente en las últimas décadas del siglo XX (Kopnina y ShoremanOuimet 2011). Este nuevo tema de investigación surgió producto del encuentro etnográfico de los antropólogos con las iniciativas de conservación en aquellas zonas aisladas donde habitaban los grupos que tradicionalmente interesaban a la disciplina (Orlove y Brush 1996).

Los estudios iniciales se concentraron en la descripción de las adaptaciones materiales de los grupos humanos a las nuevas condiciones creadas por las áreas protegidas (APs), utilizando perspectivas teóricas y metodológicas de la antropología ecológica y la ecología humana (Marks 1976). Especial relevancia tuvieron los estudios sobre sistemas de manejo de recursos de uso común entre pescadores y otros grupos locales, al demostrar su capacidad de autoorganización para crear sistemas de manejo de los recursos naturales (Berkes 1985; Acheson 1981; McCay 1978).

Con la consolidación de la disminución de la biodiversidad como problema ambiental de carácter global durante las décadas de 1980 y 1990, las investigaciones comenzaron a ampliar sus horizontes teóricos, integrando elementos de la ecología política, la antropología del desarrollo, losestudiosculturalesylosmovimientos sociales. Estas nuevas aproximaciones buscaron comprender y construir una perspectiva crítica del proceso de globalización de los discursos y las políticas conservacionistas, así como registrar etnográficamente las respuestas políticas que los diversos grupos organizados daban a la imposición de las nuevas restricciones en el uso de los recursos naturales (Agrawal y Gibson 1999).

En este sentido, fueron determinantes para la construcción de modelos alternativos de conservación los diversos conflictos socioambientales que caracterizaron la época de expansión capitalista en la zonas de alta biodiversidad, especialmente, en la regiones tropicales (Escobar 2008). Este proceso alineó las demandas territoriales de los movimientos sociales de indígenas, afrodescendientes y campesinos con las protestas conservacionistas por la destrucción de los últimos remanentes de naturaleza prístina ${ }^{1}$. Este encuentro generó un pacto político que comprometió, por una parte, el apoyo de los agentes de la conservación en la defensa del territorio, la lucha por autonomía política y el reconocimiento étnico de los grupos locales y, por otra, el compromiso de éstos últimos de cuidar y mantener la biodiversidad (Cunha y Almeida 2001). Esta alianza sentó las bases de una ecología política de los movimientos sociales, que vio en las cosmovisiones y prácticas culturales de los grupos locales una alternativa al dominio técnico y científico sobre la naturaleza promovido por las políticas del desarrollo y reivindicó los derechos colectivos sobre el territorio (Escobar 1998).

Estas movilizaciones sociales fueron incorporadas en el establishment conservacionista,

En América Latina se destacan las experiencias de las comunidades negras del Pacífico colombiano que participaron del Proyecto de Conservación BioPacífico (Grueso et al. 1998) y la lucha de los seringueiros y de su líder Chico Mendes en la Amazonia brasileña (Arnt 1994). 
caracterizando un momento de transformación en las ideologías y prácticas de protección de la biodiversidad, que ganó una dimensión global con la proclamación de la Convención de la Diversidad Biológica de las Naciones Unidas en el año 1992. Este acuerdo internacional incorporó las demandas por justicia social de años anteriores y estableció una serie de metas, directrices y fuentes de financiamiento para incentivar y orientar las políticas públicas de conservación de la biodiversidad.

El nuevo escenario conservacionista inaugurado por la "Cumbre de la Tierra de Rio 1992" incentivó el desarrollo de iniciativas que intentaron conjugar protección de la biodiversidad con desarrollo económico y social, entre las que se destacan las experiencias de "Conservación de Base Comunitaria" y los "Proyectos de Conservación y Desarrollo Integrado" (Wells et al. 1999; Brandon et al. 1998). Estos modelos concentraron sus esfuerzos en la promoción de actividades económicas asociadas a la explotación regulada de los recursos naturales, enfocando sus intervenciones a nivel local y en el ámbito comunitario. Estas experiencias se multiplicaron en las regiones de alta biodiversidad, ampliando el tejido social de los lugares en las que fueron establecidas y modificando las relaciones de poder al interior de las comunidades (West 2006). En este sentido, las principales críticas que recibieron se asociaron a la falta de reconocimiento de los contextos sociales y políticos en los que fueron implementados, provocando, por ejemplo: la intensificación de antiguos conflictos, la formación de nuevos clivajes al interior de las comunidades: entre quienes conservan y quienes no lo hacen, el aumento de las asimetrías de poder y de las desigualdades sociales (Brechin et al. 2002; Brosius et al. 1998).
A pesar de estas críticas, la inserción de modelos que consideran y fomentan la dimensión humana de la biodiversidad permitió ampliar el debate sobre los mejores mecanismos para proteger los ecosistemas y relativizar la supuesta oposición sociedad/naturaleza que dominó el escenario conservacionista de años anteriores (Ferreira 2004).

En la actualidad se reconoce que la preocupación por el estado y protección de la biodiversidad se ha ampliado a diferentes segmentos $y$ actores sociales; desde las grandes ONGs que promueven la protección de especies y hábitats emblemáticos a nivel mundial, pasando por las agencias nacionales de medio ambiente que crean áreas protegidas con el objetivo de cumplir las metas internacionales, hasta los grupos locales que promueven la protección de espacios naturales o sistemas de recursos naturales en sus localidades y áreas de trabajo (Bryant 2009; Ferreira et al. 2007).

Existe consenso entre los tomadores de decisión acerca de la necesidad de aumentar el número y cobertura de áreas protegidas a través de mosaicos o redes de APs que se integren en las dinámicas del paisaje, combinando iniciativas de conservación que permitan el uso sustentable de los recursos con unidades de conservación de protección estricta que resguarden la integridad y funcionamiento de los ecosistemas (Laffoley et al. 2008). Esta nueva perspectiva supera la visión de las áreas protegidas como islas de biodiversidad ${ }^{2}$, incorporando la preocupación

Este modelo asume que la función de las Áreas Protegidas es preservar zonas de naturaleza prístina intocadas por el hombre o wilderness según el padrón anglosajón. Esta perspectiva se desarrolló con fuerza en los Estados Unidos y caracterizó la conservación de la biodiversidad en su etapa inicial, a través de la creación de Parques Nacionales como el Parque de Yellowstone en 1872 . 
por su conectividad con el paisaje y su función en la mantención de los servicios ambientales que un determinado ecosistema provee (Wiens 2009). Los últimos avances respecto de los modelos y estrategias de conservación dicen relación con la integración de la dimensión adaptativa de los sistemas socio-ecológicos y el papel que las APs pueden cumplir para promover su resiliencia frente a los impactos del cambio global (Palomo et al. 2014).

El escenario actual de la conservación de la biodiversidad exige que se integren nuevos problemas y preguntas de investigación (Mace 2014). Así, por ejemplo, en el ámbito político, la creación de APs de gran tamaño y la aplicación de la perspectiva ecosistémica en el manejo de los recursos naturales orienta las indagaciones hacia el estudio de sistemas de gobernanza a escala regional y trasnacional (Leenhardt et al. 2013). En la dimensión cultural, el reconocimiento de la relación entre lo global y lo local exige prestar atención acerca de las múltiples conexiones y fricciones entre conocimientos, espacios y actores sociales promovidos por la conservación de la biodiversidad, y su papel en la rearticulación de categorías como naturaleza, lugar, territorio y desarrollo (Escobar 2008; Tsing 2005). En el plano económico, la valorización de los servicios ecosistémicos asociados a las APs levanta la interrogante sobre la participación de las poblaciones locales en la repartición de estos beneficios (Sommerville et al. 2010). En suma, una serie de problemas y preguntas que subrayan el vigor de la conservación de la biodiversidad como tema de investigación y la pertinencia de la perspectiva antropológica para su comprensión.

\section{Conservación marina: la expansión de las políticas de regulación y cuidado de la biodiversidad en los océanos a nivel global}

Los primeros antecedentes sobre el cuidado de especies y la protección de áreas costeras pueden encontrarse en algunas prácticas y conocimientos tradicionales de diversos grupos indígenas, preferentemente en Oceanía y Asia (Gadgil et al. 1993). A pesar de que el sentido de estas prácticas no se relaciona directamente con lo que hoy conocemos como conservación de la biodiversidad, es posible asegurar que el manejo de los recursos naturales; por medio de la identificación de áreas de exclusión de pesca, la prohibición de actividades de explotación en épocas específicas del año y la elaboración de artes de pesca que permiten seleccionar los individuos, es una práctica arraigada entre los grupos humanos especializados en la explotación de los recursos del mar (Acheson 1981; Berkes 1977).

Oficialmente, se reconoce que la disminución de las pesquerías en los países desarrollados durante las décadas de 1950 y 1960, producto de la sobrepesca, motivó el establecimiento de los primeros acuerdos internacionales para regular la explotación de los recursos pesqueros y sentar las bases para el desarrollo de la conservación marina a nivel global: el Programa de Mares Regionales de la ONU del año 1974 y la Convención de los Derechos del Mar del año 1982 (Toropova et al. 2010).

Hoy existe consenso en el mundo científico acerca de la magnitud de la crisis ambiental de los océanos y la urgente necesidad de establecer medidas para mitigar los impactos producidos por la acción humana en los ecosistemas (Halpern et al. 2008). La drástica 
disminución de la biodiversidad y la reducción de las capturas pesqueras observadas en los últimos años han sido las manifestaciones palpables de esta crisis, cuya intensidad parece ir en aumento producto del cambio climático (Doney et al. 2012).

Entre las iniciativas para enfrentar este problema ambiental se encuentran las Áreas Marinas Protegidas (AMPs), definidas como:

un espacio geográfico claramente definido, reconocido, dedicado y manejado, a través de medios legales u otros medios efectivos, para lograr la conservación en el largo plazo de la naturaleza con sus servicios ambientales y valores culturales asociados (Dudley 2008:8) [traducido por el autor].

Esta definición reúne una gran diversidad de categorías de protección; desde aquellas de protección estricta, como los Parques Nacionales Marinos, que no permiten usos productivos de los recursos naturales, hasta las que permiten su uso sustentable y regulado, como las Áreas Marinas Protegidas de Múltiples Usos.

Se ha reconocido internacionalmente que las AMPs generan beneficios para: la conservación y restauración de ecosistemas marinos degradados, la protección de especies emblemáticas, el aumento de los stocks pesqueros, la recuperación de pesquerías artesanales, la diversificación económica de las comunidades locales y el empoderamiento político de los actores sociales (Christie et al. 2003; Halpern y Warner 2002; Agardy 1994)

En los últimos años la creación de AMPs se ha intensificado considerablemente en todos los océanos del mundo, transformándose en el instrumento de mayor impacto para reducir la pérdida de la biodiversidad y asegurar la protección de los ecosistemas marinos (Thorpe et al. 2011). Los datos oficiales reunidos en el Atlas de Protección Marina ${ }^{3}$ indican que en el año 2017 un 3,08\% de la superficie marina mundial se encuentra protegida

A nivel internacional, esta tendencia es el resultado del proceso de institucionalización de la conservación de la biodiversidad promovida, principalmente, por la incorporación en las políticas públicas nacionales de las Metas de la Convención de la Diversidad Biológica $(C D B)^{4} y$ los Objetivos de Desarrollo Sostenible (ODS) ${ }^{5}$.

A pesar de los incipientes avances en el número y cobertura de las AMPs, la constatación del fracaso en la implementación y gestión de muchas de éstas áreas, así como los impactos negativos provocados en las poblaciones locales, han generado un creciente interés entre científicos y tomadores de decisión por

3 Disponible en: www.mpatlas.org/explore/. Consultado en 24 oct. 2017.

4 Las metas de conservación surgieron como un mecanismo para promover la concretización de los objetivos del "Convenio sobre la Diversidad Biológica" (CDB) y monitorear su estado de avance. En 1995 Chile ratificó el CDB comprometiéndose a cumplir las metas propuestas por este acuerdo internacional. En la Conferencia de las Partes del CDB del año 2010 (COP-10) fue establecido el Plan Estratégico para la Diversidad Biológica durante el período 2011-2020 que contempla veinte metas, denominadas Metas de Aichi. La Meta 11 de Aichi establece que para el año 2020 se debe conservar efectivamente por lo menos el $10 \%$ de los océanos del mundo a través de Áreas Marinas Protegidas. Por su parte, la Meta 17 establece que para el año 2015 las partes deberán haber elaborado, adoptado e implementado las Estrategias y Planos de Acción para la conservación efectiva, participativa y actualizada de la biodiversidad. Disponible en: www.cbd.int/sp/targets/. (consultado en febrero del 2016).

5 Acuerdo internacional firmado el año 2015 por los países miembros de la ONU con el objetivo de erradicar la pobreza, proteger el planeta y asegurar la prosperidad, estableciendo una nueva agenda de desarrollo para el período 2015-2030. Esta nueva agenda global se compone por 17 Objetivos o Metas que incluyen un objetivo dedicado exclusivamente al cuidado y protección de los Océanos. Disponible en: http://www.un.org/sustainabledevelopment/es/ oceans/ (consultado en febrero del 2016). 
comprender la complejidad de las dimensiones humanas de la conservación marina (Christie et al. 2003).

De esta forma, recientes investigaciones a nivel internacional han apuntado a la importancia de las dinámicas institucionales locales en la gestión de las áreas protegidas (Christie et al. 2009), el reconocimiento de los conflictos entre diversos actores sociales y los mecanismos para su resolución (Bavinck y Vivekanandan 2011), los imaginarios culturales e ideologías construidas en torno del patrimonio natural protegido (Fraga 2005), los aportes del conocimiento indígena al manejo de recursos naturales (Nursey-Bray 2011), las transformaciones en las relaciones de género y el papel de las mujeres en las organizaciones locales asociadas a las iniciativas de conservación marina (Frangoudes et al. 2008).

Estos estudios demuestran que la conservación marina se está extendiendo por los océanos del mundo, transformando las dinámicas sociales construidas en torno de los recursos naturales marino-costeros y generando un nuevo campo de relaciones entre naturaleza, cultura y sociedad.

\section{Convergencias y desafíos: el aporte de la antropología a la conservación marina en Chile}

En Chile los estudios antropológicos enfocados en la conservación de la biodiversidad son reducidos y limitados a experiencias particulares. En general, los trabajos responden al esfuerzo de investigadores que han concentrado sus estudios en algunas zonas específicas del país, como el sur austral o el norte grande, y cuyo interés en la conservación de la biodiversidad se deriva de temas consolidados en la antropología nacional, como los etnoconocimientos de pueblos indígenas sobre flora y fauna o la relación naturaleza y cultura en comunidades locales.

Entre ellos se destacan los trabajos de Castro y Romo (2008) sobre etnoconocimientos, manejo de recursos naturales y su conservación en el norte de Chile, los estudios sobre conservación biocultural y ética ambiental en la Patagonia chilena de Rozzi et al. (2003), y una serie de recientes investigaciones dedicadas específicamente a las dimensiones humanas de la conservación de la biodiversidad y/o espacios naturales (Araos y Ther 2017; Araos et al. 2017; Libuy 2016; Catalán 2015; Araos 2014; Serra 2012; Araos y Ferreira 2013; Solari et al. 2012; Skewes et al. 2012, 2014; Álvarez y Navarro 2010; Recasens 2005).

Estas últimas investigaciones han mostrado con mayorprecisiónlas diferentesformasqueasumen las dimensiones humanas de la conservación. Se han concentrado en la descripción y análisis de los usos tradicionales de los recursos naturales y los impactos que el proceso de modernización capitalista ha provocado en los modos de vida de las poblaciones locales. Los estudios han demostrado que la conservación emerge como un fenómeno ambivalente, ya sea como una alternativa de desarrollo local sustentable generada a través de acciones colectivas que buscan ejercer una ciudadanía plena, o como una nueva restricción/imposición a las actividades económicas de los habitantes de las localidades afectadas por la creación de las áreas protegidas. En este sentido, el carácter que asume la conservación depende de la capacidad de apropiación local de los 
objetivos y discursos conservacionistas y de su resignificación a partir de los usos locales de los recursos y los espacios naturales.

En esta línea, los trabajos antes mencionados de los antropólogos Serra y Araos muestran un interesante contrapunto en el entramado sociopolítico del proceso de establecimiento exógenoytop-down y/ola emergencia endógena y bottom-up de Áreas Protegidas Marinas en la zona costera de Chile. Los estudios subrayan, por una parte, las deficiencias en la gestión de las AMPs establecidas sin participación social y los conflictos provocados por la desconsideración de los usos históricos de los recursos naturales de las comunidades indígenas, como fue el caso de la Área Marina Protegida de Múltiples Usos Lafquen Mapu Lahual en la zona costera de la Provincia de Osorno (Serra 2012). Sin embargo, por otra parte, destacan el papel que las AMPs pueden cumplir como alternativas para la protección de los territorios, la mantención de los modos de vida de las comunidades de pescadores artesanales y el ejercicio de la descentralización en la toma de decisiones ambientales, ejemplificado por la experiencia de conservación marina municipal en la comuna de Navidad (Araos y Ferreira 2013).

A estos trabajos pioneros para la antropología de la conservación marina en Chile habría que, necesariamente, sumar los esfuerzos de otras disciplinas. Cabe destacar el trabajo de diversos biólogos dedicados al manejo de recursos naturales y sus interfaces con la conservación. Entre éstos se destacan los trabajos de Castilla (1986), Fernández y Castilla (2005), Hucke et al. (2010), Jorquera-Jaramillo et al. (2012), Squeo et al. (2012), Gelcich et al. (2013, 2015), Gaymer et al. (2014), Oyanedel et al. (2016). Investigaciones que exponen los beneficios del establecimiento de Áreas Marinas Protegidas para la sustentabilidad de los recursos marino-costeros y la importancia de generar mecanismos de gestión adecuados para cada contexto ecológico y social.

El desafío que la conservación marina está colocando a la antropología y a otras disciplinas interesadas en las dimensiones humanas es complejo y crece día a día con la creación de nuevas AMPs en el mar chileno. En la actualidad existen 34 AMPs en la Zona Económica Exclusiva de Chile y su mar territorial, que cubren una superficie aproximada de $1.000 .000 \mathrm{~km}^{2}$ y que representan el 29\% del área marina total del país. En los últimos años las Áreas Marinas Protegidas se han expandido en número y cobertura, alcanzando las metas internacionales y transformando al país en unos de los líderes de conservación marina en el mundo. Estos avances se explican, principalmente, por la creación de AMPs de gran tamaño en las zonas adyacentes a las Islas Oceánicas chilenas. Así, por ejemplo, en el año 2010 se creó el Parque Marino Motu Motiro Hiva en la Isla de Sala y Gómez que protege 150.000 km², en el 2015 se creó el Parque Marino Nazca-Desventuradas en la zona marina adyacente a las Islas San Félix y San Ambrosio que protege $297.528 \mathrm{~km}^{2}$, entre 2016 y 2017 dos AMPs en el archipiélago de Juan Fernández que protegen aproximadamente $450.000 \mathrm{~km}^{2}$, y durante el año 2018 se espera el decreto oficial de creación de una gran AMP en la zona adyacente a la Isla de Pascua de aproximadamente $700.000 \mathrm{~km}^{2}$. Con estas cifras Chile tendrá en el año 2018 el 48\% de su mar protegido (en torno de $1.600 .000 \mathrm{~km}^{2}$ ).

No obstante, estos indudables avances en la cobertura y extensión de las AMPs, la representatividad de los ecosistemas, la 
distribución geográfica y el nivel de protección muestran importantes lagunas (MMA, 2015). Estos elementos representan la visión oficial/ gubernamental acerca de los principales desafíos para la política pública de conservación marina, asociados en su mayoría a la definición de un Sistema Nacional de Áreas Protegidas y la creación del Servicio de la Biodiversidad y Áreas Protegidas.

Sin embargo, la revisión de la experiencia internacional sobre las dimensiones humanas de la conservación marina y su propio desarrollo histórico en Chile ponen en evidencia la necesidad de incorporar nuevos desafíos y demandas, asociados, en su conjunto, a una gestión de la conservación que sea inclusiva socialmente y apropiada a cada contexto territorial.

Específicamente, referimos a: i) comprender y reconocer el papel de los conflictos sociales asociados al uso y manejo de los recursos naturales propiciados 0 transformados por la creación de áreas marinas protegidas; ii) construir una perspectiva crítica del proceso de toma de decisiones que define la creación y administración de las áreas marinas protegidas; iii) aprehender la relaciones entre cultura, naturaleza y sociedad que la conservación de la biodiversidad está generando entre diversos actores sociales; iv) registrar y divulgar acciones colectivas de protección del patrimonio natural y cultural de las comunidades locales.

Estos desafíos implican desarrollar una alternativa teórica y metodológica que, a partir de una perspectiva antropológica, permita dialogar con otras disciplinas y participar en la construcción de las políticas públicas de conservación de la biodiversidad en el país.

\section{Arenas, actores y toma de decisiones: una propuesta analítica para el estudio de las dimensiones humanas de la conservación marina}

La propuesta analítica asume una estrecha relación entre los conflictos y la toma de decisiones, entendiéndola como un proceso de constante negociación entre actores con intereses diferentes y contradictorios sobre los recursos naturales. A pesar de la visión negativa que se puede asumir sobre los conflictos, por ejemplo, como desencadenantes de actos de violencia, extraemos de ellos su potencia transformadora; como formas de sociabilidad (Simmel 1983) que promueven acciones colectivas con el objetivo de formar nuevas organizaciones o bien para reconfigurar las ya existentes (Ferreira 2007).

Por lo tanto, el foco inicial del proceso de análisis se concentra en los conflictos por los usos de los recursos naturales provocados por la creación de áreas protegidas y el proceso de toma de decisiones que define su establecimiento. Para analizar esta relación se utiliza el concepto de arena, que remite al espacio de interacción sociopolítica donde se toman las decisiones (Araos y Ferreira 2013). Este concepto se basa en corrientes teóricas que destacan el papel de los actores y sus interacciones en la conformación del orden social, como el neo-institucionalismo de Ostrom (1990), el constructivismo ambiental de Hannigan (1995), la sociología del riesgo de Renn (2008) y la sociología del desarrollo de Long (2001). Tal y como este último autor señala, es en las arenas donde ocurren las luchas y negociaciones sobre los significados y prácticas de la vida social, congregando en su interior a actores con diferentes recursos, intereses, valores culturales y trayectorias políticas (Long 2004). 
Asumiendo estos antecedentes es posible reconocer que las principales características de las arenas son: i) la presencia de múltiples actores sociales; ii) la interacción de los actores a partir de posiciones flexibles y en constante cambio; iii) el desarrollo de acciones estratégicas; iv) la movilización de recursos para influir en la toma de decisiones; v) la construcción intersubjetiva de marcos de interpretación comunes.

Este sistema de interacción puede materializarse en diferentes y diversas situaciones de acción en las que es posible observar la movilización de recursos económicos, políticos o culturales por parte de los actores con la intención de influir en la toma de decisiones. Este proceso va definiendo diferentes reglas en el uso de los recursos naturales que, en la medida en que se entraman en la vida política y social, se transforman en instituciones que regulan los diversos intereses sobre la biodiversidad. Esta perspectiva permite comprender el modo de funcionamiento de las diversas reglas que componen el sistema de gobernanza de los recursos naturales en una determinada realidad y los mecanismos institucionales de permanencia y transformación que lo caracterizan (Ostrom 2005).

Una estrategia sociológica para abordar la interacción social que se despliega en las arenas es el análisis de redes sociales (ARS). Esta perspectiva pone el acento en las relaciones de los actores más que en sus atributos, permitiendo identificar la estructura de una determinada agregación social -densidad, cohesión, dispersión - y el papel jugado por algunos actores para la movilización social de la red - brokers o puentes - (Bodin et al. 2006).

No debe perderse de vista que, a pesar de ser posible identificar los contornos específicos de una determinada arena y las decisiones tomadas en su interior, los fenómenos socioambientales contemporáneos se configuran en torno de múltiples y diversas arenas decisorias interconectadas entre sí, siendo necesario circular por las diferentes escalas en movimientos de descenso y ascenso analítico que permitan comprender la complejidad del proceso estudiado (Morán 2011).

En este sentido, se concuerda con Escobar (2008) que la conservación de la biodiversidad forma parte de un amplio y complejo proceso de articulación de fuerzas globales y locales que resultan en nuevos sistemas de naturaleza y cultura. Por lo tanto, acreditamos que la incorporación, negociación y apropiación local de la conservación de la biodiversidad se define en términos de conexiones y fricciones entre flujos de informaciones globales/universales, como la biodiversidad, y locales, como los usos y conocimientos sobre los recursos naturales (Tsing 2005). La relevancia de las fricciones es que tienen el potencial de generar nuevos arreglos de cultura y poder, que pueden reorientar las trayectorias globales y ampliar las posibilidades locales (Araos et al. 2014).

Esta perspectiva analítica implica la identificación certera de los niveles y escalas de análisis; desde lo micro social a nivel local hasta lo macro social a nivel global, desde sistemas ecológicos limitados a pequeñas bahías 0 zonas estuarinas hasta extensas eco-regiones marinas, desde administraciones municipales hasta sistemas de gobernanza transfronterizos. El reconocimiento inicial del nivel y escala de análisis permitirá, posteriormente, establecer las conexiones con los procesos que se dan en los otros niveles, agregando complejidad a la indagación. Así, haciendo una analogía con 
los Sistemas de Información Geográfica (SIG), en la medida en que se agregan nuevos shapes se va construyendo un sistema dinámico de observación y análisis que permite aprehender las transformaciones en los diversos usos de los recursos naturales propiciados por la creación de áreas protegidas y las conexiones del proceso de toma de decisiones.

En la Figura 1 se ilustra el modelo de análisis de los procesos de toma de decisiones ambientales propuesto en este trabajo. El modelo toma como punto de partida el análisis del sistema de interacción social desarrollado en torno a una arena específica, como puede ser la creación de una AMP en una determinada zona costera, a partir de la identificación de la red social que se despliega en su interior. Una vez que se establecen sus contornos, estructura y las acciones colectivas movilizadas, se identifican sus conexiones con otras arenas o espacios de gobernanza. Esta relación puede darse a través de actores específicos, de canales institucionales formales para acceder a otros niveles políticos, de la acción colectiva de movimientos sociales o bien del escalamiento de un conflicto social. A partir de aquí, se incorpora la dimensión escalar de los fenómenos ambientales contemporáneos - tiempo, espacio, ecosistema, organización social y administrativa - buscando

Figura 1. Modelo de análisis para la toma de decisiones ambientales

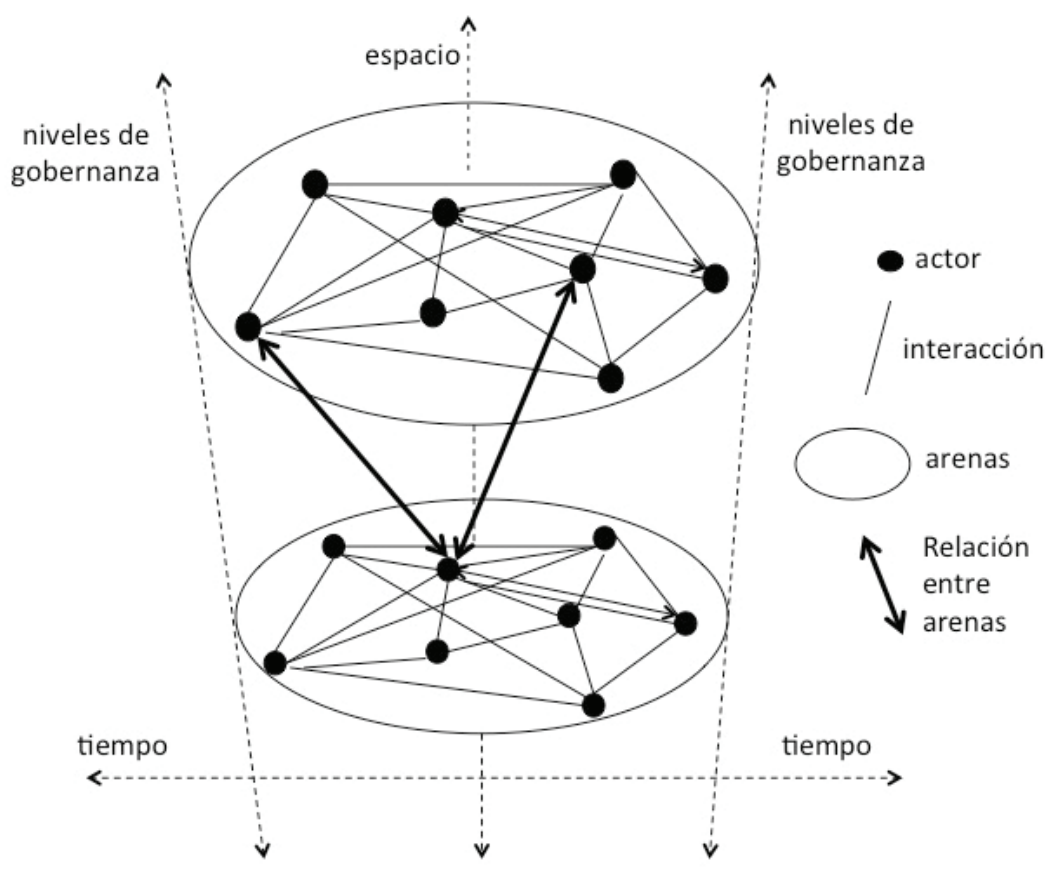

Fuente: Elaborado por el autor, adaptado de Calvimontes 2013. 
comprender la conexión entre procesos de toma de decisiones experimentados en diferentes niveles del sistema de gobernanza.

Metodológicamente, la propuesta gana densidad al incorporar cuatro instrumentos analíticos: i) la descripción de situaciones de acción en las que se manifiestan los intereses sobre la biodiversidad y se movilizan recursos con el objetivo de influir en las decisiones, por ejemplo, en las reuniones de los comités o mesas de gestión de las APs; ii) etnografías multisituadas de los diversos actores que participan en el proceso de creación y gestión de APs, por ejemplo, Líderes Comunitarios, Fundaciones, ONGs, Científicos; iii) análisis de redes sociales que describen y analizan las interacciones y posiciones de los diversos actores; iv) las trayectorias institucionales de la conservación y uso de los recursos naturales de una determinada unidad de análisis.

En suma, se aboga por impulsar una agenda de investigación antropológica que dialogue críticamente con la expansión de la conservación marina en la zona marino-costera del país y que exponga la complejidad que envuelve la creación y gestión de Áreas Marinas Protegidas. Este desafío implica, finalmente, asumir la certera pregunta que Arturo Escobar hacía a mediados de la década del noventa, cuando los proyectos de conservación se consolidaron en los ecosistemas tropicales de América Latina: whose knowledge? whose nature?, ¿de quién es el conocimiento que fundamenta la conservación?, ¿a quién pertenece la naturaleza protegida? Preguntas sin una respuesta final, mas que indican una forma de hacer las cosas, advierten de la necesidad de democratizar el proceso de toma de decisiones y de entender a la conservación como un pacto social entre actores diferentes y desiguales.

\section{Conclusión: contribuciones a las políticas públicas de conservación marina}

Este artículo procuró destacar el carácter político de la conservación de la biodiversidad y la necesidad de incorporar y complejizar la dimensión humana. Esto implica ir más allá de la identificación de modelos o categorías de áreas protegidas que incorporen el uso regulado de los recursos naturales, mas bien, pone el acento en la consideración del proceso que determina la creación y gestión de una área protegida y del entramado de intereses, conflictos y alianzas que caracterizan la toma de decisiones.

En este sentido, asumimos que el principal desafío para la nueva política de conservación marina en Chile es el establecimiento de un sistema de gobernanza flexible, descentralizado, democrático y transparente, que incluya la diversidad socio-territorial y que sea capaz de adaptarse a las nuevas condiciones ambientales.

Para esto es importante que la creación de AMPs considere y respete los usos locales de los recursos naturales, transformando las prácticas insustentables e incorporando los sistemas tradicionales de manejo.

Asimismo, es relevante que la gestión de las AMPs incluya mecanismos formales de explicitación y resolución de conflictos de manera permanente, que promuevan la deliberación pública y orienten las regulaciones en el manejo de los recursos naturales.

De esta forma, se reconoce que la conservación de la biodiversidad dejó de ser una cuestión de expertos, sean éstos de la academia, el gobierno o las ONGs, que definían el desarrollo de la conservación a través de evaluaciones técnico-científicas, para ampliarse hoy a todos 
los segmentos de la sociedad. Un nuevo punto de partida que incentiva establecer acuerdos de convivencia entre los actores con el objetivo de proteger la integridad de los ecosistemas y asegurar el bienestar humano.

Para la antropología este nuevo tema de investigación implica renovar las perspectivas teóricas y metodológicas, abriéndose a la interdisciplinariedad y el diálogo crítico con las otras ciencias del campo socio-ambiental.

Específicamente, el desarrollo de la antropología delaconservación debereafirmar su compromiso con las comunidades que tradicionalmente han interesado a los antropólogos, como los pueblos indígenas, las comunidades rurales y litorales, pero asumiendo que la práctica conservacionista involucra actores diversos y con intereses diferentes sobre los recursos naturales protegidos. En este sentido, se torna esencial analizar la manera en que esos actores negocian y toman decisiones, destacando las asimetrías de poder y las estrategias de acción desarrolladas. En este punto es relevante identificar los imaginarios construidos en torno de la naturaleza protegida, con el fin de comprender los usos de los recursos naturales que serán restringidos y/o regulados por la creación de áreas protegidas y sus implicaciones para la gestión de la biodiversidad. Sin duda, esta es una cuestión relegada a segundo plano en cualquier diagnóstico o informe técnico, pero es central al momento de establecer puntos de encuentro entre los actores que permitan generar pactos de convivencia entre los usuarios.

Finalmente, es imprescindible que los estudios antropológicos sobre la conservación continúen identificando y relevando la diferencia, mostrando otras maneras de hacer las cosas, reconociendo la capacidad de los grupos humanos de generar prácticas de uso sustentable de los recursos naturales.

\section{Bibliografía}

Acheson, J.M. 1981. "Anthropology of fishing". Annual Review of Anthropology 10: 275-316.

Agardy, T. 1994. "Advances in marine conservation: the role of marine protected areas". Trends in Ecology and Evolution 9: 267-270.

Álvarez, R. y Navarro, M. 2010. "Conflictos asociados a los múltiples uso". Conservando el Mar de Chiloé, Palena y las Guaitecas. Síntesis del Estudio Investigación para el Desarrollo de Área Marina Costera Protegida Chiloé, Palena y Guaitecas. HuckeGaete, R., Lo Moro,P y Ruiz, J. (Eds.) Puerto Montt: Gobierno Regional de Los Lagos. 125-143.

Araos, F. y Ther, F. 2017. "How to adopt an inclusive development perspective for marine conservation: Preliminary insights from Chile". Current Opinion in Environmental Sustainability 24 :68-72.

Araos, F. et al. 2017. "Conservación Marina y Costera en Chile: trayectorias institucionales, innovaciones locales y recomendaciones para el futuro". Clima de Tensão. Ação humana, biodiversidade e mudanças climáticas, Ferreira, L. et al. (Eds.)
Campinas: Editora UNICAMP. 529-544.

et al. 2014. 'Pre', step-zero and beyond: local paths in marine conservation in Latin America. 2nd World Small-Scale Fisheries Congress, Mérida, México, September.

2014. Para além da Biodiversidade: Dimensões Humanas da Conservação Marinha em duas Regiões da América do Sul. Tese para obter o título de Doutor em Ambiente e Sociedade, Núcleo de Estudos e Pesquisas Ambientais (NEPAM), Instituto de Filosofia e Ciências Humanas (IFCH), Universidade Estadual de Campinas (UNICAMP), Campinas, Brasil.

y L.C. Ferreira, L.C. 2013. "The construction of an environmental arena for marine conservation in Chile". Ambiente $e$ Sociedade 16(3): 119-136.

Arnt, R. (Ed.). 1994. O Destino da Floresta. Reservas Extrativistas e Desenvolvimento Sustentável na Amazônia. Rio de Janeiro: Ed. Relume Dumará.

Agrawal, A y Gibson, C. 1999. "Enchantment and disenchantment: The role of communuty in Natural resource 
conservation". World Development 27(4): 629-649.

Bavinck, M. y Vivekanandan, V. 2011. "Conservation, conflict and the governance of fisher wellbeing. Analysis of the establishment of the Gulf of Mannar National Park and Biosphere Reserve". Environmental Management 47(4): 593-602.

Berkes, F. 1985. "Fishermen and "The tragedy of the commons"”. Environmental Conservation 12(3): 199-206.

1977. "Fisheries resource use in a subantartic indian community". Human Ecology 5(4): 289-307.

Bodin, Ö, Beatrice, C. y Ernstson, H. 2006. "Social networks in natural resource management: What is there to learn from a structural perspective?" Ecology and Society 11(2): r2.

Brandon, K., Redford, K y Sanderson, S. (Eds.). 1998. Parks in Peril: People, politics, and protected areas. Covelo, CA, Washington, D.C.: Island Press.

Brechin S.R. et al. 2002. "Beyond the square wheel: toward a more comprehensive understanding of biodiversity conservation as social and political process". Society and Natural Resources 15(1): 41-64.

Brosius J.P., Tsing, A y Zerner, C. 1998. "Representing communities: Histories and politics of community-based natural resource management". Society and Natural Resources 11:157-168.

Bryant, R.L. 2009. "Born to be wild? Non-governmental organisations, politics and the environment". Geography Compass 3: 1541-1558.

Calvimontes, J. 2013. Bandidos na Serra do Mar? Conflitos, estratégias e usos múltiplos dos recursos naturais na MataAtlântica, São Paulo. Tese para obter o título de Doutor em Ambiente e Sociedade, Núcleo de Estudos e Pesquisas Ambientais (NEPAM), Instituto de Filosofia e Ciências Humanas (IFCH), Universidade Estadual de Campinas (UNICAMP), Campinas, Brasil.

Castilla, J.C. 1986. "¿Sigue existiendo la necesidad de establecer parques y reservas marítimas en Chile?". Ambiente y Desarrollo 2: 53-63.

Castro, V. y Romo, M. 2008. "Tradiciones Culturales y Biodiversidad". Biodiversidad de Chile: Patrimonio y Desafíos. 2 edición. Santiago: Comisión Nacional del Medio Ambiente. 468-493.

Catalán, E. 2015. Relaciones humano-ambiente en el Parque Nacional La Campana: Una trayectoria de encuentros y desencuentros entre Comunidades Locales y el Área Protegida. Memoria para optar al título profesional de Antropóloga Social. Departamento de Antropología, Universidad de Chile, Santiago.

Christie, P. et al. 2009. "Back to basics: An empirical study demonstrating the importance of local-level dynamics for the success of tropical marine ecosystem-based management". Coastal Management 37(3-4): 349-373.

., Mccay, B.J. y Miller, M.I. 2003. "Toward developing a complete understanding: a social science research agenda for marine protected areas". Fisheries 28(12):22-26.

Cunha, M. y Almeida, M.W.B. 2000. "Indigenous people, traditional people, and conservation in the Amazon". Daedalus. Journal of the American Academy of Arts and Sciences 129(2): 315-338.
Deguignet, M. et al. 2014. 2014 United Nations List of Protected Areas. Cambridge, UK.: UNEP-WCMC.

Doney, S.C. et al. 2012. "Climate Change Impacts on Marine Ecosystem". Annual Review of Marine Science 4: 11-37.

Dudley, N. (Ed.). 2008. Guidelines for Applying Protected Area Management Categories. Gland, Switzerland: IUCN.

Escobar, A. 2008. Territories of Difference. Place, Movements, Life, Redes. Durham: Duke Unversity Press.

1998. "Whose knowledge, whose nature? biodiversity, conservation and the political ecology of social movements". Journal of Political Ecology 5: 53-82.

Feeny, D. et al. 1990. "The tragedy of the commons: twentytwo years later". Human Ecology 18(1):1-19.

Fernández, M. y Castilla, J.C. 2005. "Marine conservation in Chile: historical perspective, lessons, and challenges". Conservation Biology 19(6):1752-1762.

Ferreira, L. et al. 2007. "Encontro das águas: dinâmicas sociais e biodiversidade na Amazônia brasileira". Teoría \& Pesquisa 50 16(1):15-37.

2004. "Dimensões humanas da biodiversidade. Mudanças Sociais e Conflitos em Torno de Areas Protegidas no Vale do Ribeira, SP, Brasil". Ambiente e Sociedade 7(1):5-29.

Fraga, J. 2006. "Local perspectives in conservation politics: the case of the Ría Lagartos Biosphere Reserve, Yucatán, México". Landscape and Urban Planning 74(3-4):285-295.

Frangoudes, K., Marugán-Pintos, B. y Pascual-Fernández, J.J. 2008. "From open access to co-governance and conservation: The case of women shellfish collectors in Galicia (Spain)". Marine Policy 32(2): 223-232.

Gadgil, M., Berkes, Fy Folke, C. 1993. "Indigenous knowledge for biodiversity conservation". AMBIO 22(2-3):151-156.

Gaymer, C.F. et al. 2014. "Merging top-down and bottom-up approaches in marine protected areas planning: experiences from around the globe". Aquatic Conservation: Marine and Freshwater Ecosystems 24(S2): 128-144.

Gelcich, S., et al. 2015. "Alternative strategies for scaling up marine coastal biodiversity conservation in Chile". Maritime Studies 14:1-13.

. et al. 2013. "Financing marine protected areas through visitor fees: insights from tourists willingness to pay in Chile". AMBIO 42(8):975-984.

Grueso L., Rosero, C. y Escobar, A. 1998. "The process of black community organizing in the southern pacific coast region of Colombia". Cultures of Politics/Politics of Cultures: Re-visioning Latin American Social Movements. Alvarez, S., Dagnino, E. y Escobar, A. (Eds.). Oxford: Westview Press. 196-219.

Halpern, B. et al. 2008. "A global map of human impact on marine ecosystems". Science 319(5865): 948-952.

y Warner, R. 2002. "Marine reserves have rapid and lasting effects". Ecology Letters 5(3): 361-366.

Hannigan, J. 1995. Environmental Sociology: a social constructionist perspective. New York: Routledge. .

Hucke-Gaete, R., Lo Moro, P. y Ruiz, J. (Eds.). 2010. 
Conservando el Mar de Chiloé, Palena y las Guaitecas. Síntesis del estudio Investigación para el desarrollo de Área Marina Costera Protegida Chiloé, Palena y Guaitecas. Puerto Montt: Gobierno Regional de Los Lagos.

Jorquera-Jaramillo C. et al. 2012. "Conservación de la biodiversidad en Chile: Nuevos desafíos y oportunidades en ecosistemas terrestres y marinos costeros". Revista Chilena de Historia Natural 85(3): 267-280.

Kopnina, H. y Shoreman-Ouimet, E. (Eds.). 2011. Environmental Anthropology Today. New York: Routledge.

Laffoley, D., Gjerde, K. y Wood, L. 2008. "Progress on marine protected areas since Durban and future directions". Parks 17(2):13 -22

Leenhardt, P.B. et al. 2013. "The rise of large-scale marine protected areas: conservation or geopolitics?". Ocean \& Coastal Management 85:112-118.

Libuy, M. 2016. Prácticas y representaciones vinculadas al bosque nativo en Aysén en el actual contexto de mercantilización de la naturaleza. Memoria para optar al título profesional de Antropóloga Social. Departamento de Antropología, Universidad de Chile, Santiago.

Long, N. 2001. Development Sociology: Actor Perspectives. London and New York: Routledge.

2004. "Actors, interfaces, and development intervention: Meanings, purposes and powers". Development intervention: Actor and activity perspectives, Kontinen, $\mathrm{T}$ (Ed.). Helsinki: Center for Activity Theory and Developmental Work Research and Institute for Development Studies, University of Helsinki. 14-36.

Luttrell, C. et al. 2013. "Who should benefit from REDD+? Rationales and realities". Ecology and Society 18(4): 52.

Mace, G. M. 2014. "Whose conservation?" Science 345: $1558-1560$.

McCay, B. 1978. "Systems ecology, people ecology, and the anthropology of fishing communities". Human Ecology 6(4): 397422.

Marks, S.A. 1976. Large Mammals and a Brave People: Subsistence Hunters in Zambia. Seattle: University of Washington Press.

Ministerio del Medio Ambiente. 2015. Las Áreas Protegidas de Chile. Santiago: MMA, Gobierno de Chile.

Moran, E. 2011. Meio Ambiente e Ciências Sociais. Interações homem-ambiente e sustentabilidade. São Paulo: Editora Senac.

Nursey-Bray, M. 2011. "Social contexts and customary fisheries: marine protected areas and indigenous use, Australia". Environmental Management 47(4):671-683.

Orlove, B.S y Brush, S.B. 1996. "Anthropology and the conservation of biodiversity". Annual Review of Anthropology 25: 329-52.

Ostrom, E. 2005. Understanding Institutional Diversity. Princeton: Princeton University Press.

1990. Governing the Commons: The Evolution of Institutions for Collective Action. Cambridge: Cambridge
University Press.

Oyanedel, R. et al. 2016. "Establishing marine protected areas through bottom-up processes: insights from two contrasting initiatives in Chile". Aquatic Conservation: Marine and Freshwater Ecosystems 26(1):184-195.

Palomo, I. et al. 2014. "Incorporating the social-ecological approach in protected areas in the Anthropocene". BioScience 64(3): 181-191.

Recasens, A. 2005. "Cultura y biodiversidad marina". Biodiversidad Marina. Valoración de usos y perspectivas. ¿Hacia dónde va Chile?. Figueroa, E. (Ed.). Santiago: Editorial Universitaria.165-189.

Renn, O. 2008. Risk governance: coping with uncertainty in a complex world. London: Earthscan.

Rozzi, R. et al. 2003. "Conservación biocultural y ética ambiental en el extremo austral de América. Oportunidades y dificultades para el bienestar ecosocial". Globalización y Biodiversidad. Oportunidades y desafíos para la sociedad chilena. Figueroa, E. y Simonetti, J. (Eds.). Santiago: Editorial Universitaria. 51-85.

Serra, D. 2012. Implementation of a marine protected area in Chile: consequences of neglecting local socio-cultural factors. Thesis submitted for the degree of Doctor of Philosophy, University of Otago, Dunedin, New Zealand.

Simmel, G. 1983. Sociologia. São Paulo: Ática.

Skewes, J.C., Guerra, D. y Henriques, C. 2014. "Patrimonio y Paisaje: Dos formas de ensamblar naturaleza y cultura en la cuenca del Río Valdivia, Sur de Chile". Chungará 46(4): 651-668.

Rehbein, R. y Mancilla, C. 2012. "Ciudadanía y sustentabilidad ambiental en la ciudad: la recuperación del humedal Angachilla y la organización local en la Villa Claro de Luna, Valdivia, Chile". Eure 38(113): 127-145.

Solari, M.E. et al. 2012. "Historia ambiental de los archipiélagos de la Trapananda (Patagonia septentrional, Chile): desafíos para la conservación de la ballena azul". CUHSO 22(1): 115-130.

Sommerville, M. et al. 2010. "The role of fairness and benefit distribution in community-based Payment for Environmental Services interventions: A case study from Menabe, Madagascar". Ecological Economics 69(6): 1262-1271.

Squeo, F. et al. 2012. "Towards the creation of an integrated system of protected areas in Chile: achievements and challenges". Plant Ecology \& Diversity 5(2): 233-243.

Thorpe, A., Failler, P. y Bavinck, J.M. 2011. "Marine protected areas (MPAs). Special feature: editorial". Environmental Management 47(4): 519-524.

Toropova, C. et al. 2010. Global Ocean Protection: Present Status and Future Possibilities. Gland, Washington, DC and New York: IUCN WCPA.

Tsing, A. 2005. Friction: An Ethnography of Global Connection. Princeton: Princeton University Press.

West, P. 2006. Conservation is our Government Now. The Politics of Ecology in Papua New Guinea. Durham: Duke University Press. 
Igoe, J. y Brockington, D. 2006. "Parks and Peoples: The Social Impact of Protected Areas". Annual Review of Anthropology 35:251-77.

Wells, M. et al. 1999. Investing in Biodiversity: A Review of Indonesia's Integrated Conservation and Development Projects.
Washington DC: World Bank.

Westley, F. et al. 1998. "People and habitat protection". Parks 8(1): 15-28.

Wiens, J. 2009. "Landscape ecology as a foundation for sustainable conservation". Landscape Ecology 24(8): 1053-1065. 
